\title{
Production and properties towards the island of stability
}

\author{
Matti Leino \\ Department of Physics, University of Jyväskylä, PO Box 35, 40014 University of \\ Jyväskylä, Finland
}

\begin{abstract}
The structure of the nuclei of the heaviest elements is discussed with emphasis on single-particle properties as determined by decay and inbeam spectroscopy. The basic features of production of these nuclei using fusion evaporation reactions will also be discussed.
\end{abstract}

\section{Introduction}

In this short review, some examples of nuclear structure physics and experimental methods relevant for the study of the heaviest elements will be presented. The emphasis is on singleparticle properties.

Several excellent review articles on the topics of this article have appeared recently. A number of them can be found in a special volume of Nuclear Physics A (Vol. 944). Articles which deal with the topics discussed here include [1-5]. Other recent review articles include, for instance, Refs. [6-9].

\section{Nuclear structure}

Nuclear structure physics deals with details of the interactions between the protons and the neutrons constituting the nucleus of an atom. Nuclei of the heaviest elements are of particular interest since their very existence is based on quantum mechanical effects creating a barrier against fission. Also, in the heaviest nuclei, very high single particle angular momenta appear, sometimes in combination with low angular momenta, leading to interesting nuclear interactions. Both single-particle and collective phenomena are of interest. Here, emphasis will be on single-particle properties when discussing structure details. Collective phenomena, especially those involved in nuclear fission, will also be touched upon. A particularly interesting but also challenging question is the influence of nuclear structure on reaction cross sections. This problem is, however, beyond the scope of this article.

\subsection{Single-particle spectrum}

The single-particle spectrum of a nucleus determines some of its most important properties. It constitutes a critical test of a theory. As a function of energy, there appear regions of larger density of single-particle levels, so called shells, separated by energy gaps. This is the shell structure of a nucleus. The closure of a shell indicates higher than average nuclear binding and stability. One of the key objectives of the study of the heaviest nuclei, or superheavy nuclei (here also, SHN) is the shell structure and its influence on nuclear half-lives and production cross sections. 
When the structure of the nucleus is studied as a function of nuclear deformation, it is observed that energy gaps appear also at nonsphericity, albeit at different proton and neutron numbers [10]. Such phenomena persist also at high deformation, possibly leading to the creation of a fission barrier also for nuclei which according to the classical liquid drop theory would fission essentially instantaneously, i.e. those with proton number $Z$ higher than 104 or so $[1,8]$. Such shell effects or shell corrections to the ground-state mass constitute the main part of the barrier against fission. Shell-correction energies in the region of SHN can typically reach 6-8 MeV.

The heaviest nucleus in nature with closed proton and neutron shells is ${ }^{208} \mathrm{~Pb}$, a particularly tightly bound so called doubly magic nucleus. The question which has been driving the study of SHN since the 1960's is whether such nuclei exist beyond ${ }^{208} \mathrm{~Pb}$ and if so, where precisely do they appear on the chart of nuclei. This problem is still incompletely understood although presently we know that there are stabilizing nuclear shells at neutron number $N=152$ and 162 in the region of rather strongly deformed nuclei [11]. The key problem is whether spherical SHN exist. Neutron number $N=184$ is the most promising candidate based on calculations and there are strong indications that experiments have already taken us to the shores of the so called island of stability with the synthesis of elements up to proton number $Z=118$ [12].

\subsection{Results from the nobelium region}

Many nuclear structure details have been learned from the study of nuclei in the so called nobelium (No) region. This is the region around ${ }^{254}$ No which nuclide can be synthesized in the reaction ${ }^{48} \mathrm{Ca}+{ }^{208} \mathrm{~Pb}$ (two doubly magic nuclei) with the rather high production cross section of about $2 \mu \mathrm{b}$. Several other nuclei in this region also have a sufficiently high yield for spectroscopic studies in ${ }^{48} \mathrm{Ca}$ - or ${ }^{48,50} \mathrm{Ti}$-induced reactions. The present cross section limit for so called in-beam studies is around $10 \mathrm{nb}$ [4]. In-beam studies give information about nuclear dynamics, in particular from rotational properties. Apart from selected results, such experiments will not be discussed further here (see, however, e.g. the review articles $[4,13])$.

The nuclei in the No region are themselves interesting from the nuclear structure point of view. They may also give information on single-particle levels of nuclei in the spherical SHN region: Spherical single-particle orbitals split into components according to the projection $K$ of the angular momentum onto the nuclear symmetry axis. Deformation brings these levels down into the more accessible No region. Furthermore, a natural requirement on a theory which is hoped to realistically describe spherical SHN is that it also describes nuclei in the No region sufficiently well. One example of a related question is the location of the spherical proton gap. According to some calculations, if the spin-orbit interaction between the $2 f_{5 / 2}$ and $2 f_{7 / 2}$ spin-orbit partners is strong, the gap is expected at $Z=114$ while for a weaker interaction it is expected at $Z=120$ [14].

Of particular interest are the so called $K$ isomers [15]. Such relatively long-lived states are due to the presence of neutron or proton orbitals with large $K$ near the Fermi surface. Then there opens up the possibility that - for example due to pair breaking - there is at low excitation energy a state with high spin which has no decay route down except by breaking the $K$ conservation rule. This leads to strong hindrance for the decay. $K$ isomers provide information e.g. on position of single-particle states and on the pairing interaction. As an example, the lowest two-quasi particle excitations are expected at an excitation energy of $2 \Delta$ where $\Delta$ is the pair-gap parameter [4]. $K$-isomer data in the No region support the existence of deformed shell gaps at $Z=100$ and $N=152$ [16]. 
A basic question related to excited nuclear states is whether they are generated by the excitation of protons or neutrons, or both. This question may be difficult to answer. Branching ratios of transitions depopulating the state in question are sometimes helpful. An important example is the $3^{+}$state in ${ }^{254} \mathrm{No}$ at $988 \mathrm{keV}$ excitation [14]. Experimental branching ratios clearly identify it as a two-proton excitation involving the Nilsson orbitals $1 / 2^{-}[521]$ and $7 / 2^{-}[514]$. The $1 / 2^{-}[521]$ proton orbital stems from the spherical $2 f_{5 / 2}$ orbital and informs us of its energy [14].

\subsection{Decay studies}

The most important decay types in the SHN region are $\alpha$ decay and spontaneous fission although the possibility of electron capture decay which may be difficult to observe should in some cases be kept in mind. Alpha decay and spontaneous fission will be briefly discussed in the following. From the point of view of half-life calculations, $\alpha$ decay is by far the easier one of these two.

\subsubsection{Alpha decay}

The half-life $T_{\alpha}$ in $\alpha$ decay depends mainly on the decay energy $Q_{\alpha}$. A good approximation is given by the Geiger-Nuttall (or Viola-Seaborg) type of formula [17]

$$
\log _{10} T_{\alpha}=a / \sqrt{ } Q_{\alpha}+b .
$$

Here, the values of the constants $a$ and $b$ are linear functions of the proton number of the decaying nucleus.

Equation (1) works very well for ground state to ground state decays in even-even nuclei. For odd-mass and odd-odd nuclei one can talk about a hindrance for transitions between different single-particle states and define the hindrance factor $H F=T_{\exp } / T_{\text {calc }}$ where $T_{\text {exp }}$ and $T_{\text {calc }}$ are the experimental and calculated half-life, respectively. Alpha decay preferably populates that single-particle state in the daughter nucleus which has the same single-particle configuration as the parent state, i.e. $H F \approx 1$. If, for example, there is a parity change and the nucleon spin changes direction (spin flip), typically $H F>1000$. The barrier penetration probability depends strongly on the energy and in the region of SHN one $\mathrm{MeV}$ of excitation energy in the daughter nucleus typically reduces the decay constant by two to three orders of magnitude.

Systematic studies of $\alpha$-decay energies reaching over long isotope chains can give evidence of shell or sub-shell closures [17]. Combined data from both cold and hot fusion reactions (see Sect. 3) reaching from $Z=106$ to $Z=118$ show clear evidence of a shell at $N=162$ and also show a strong decrease of $\alpha$-particle energy when $N$ increases beyond 165 , an indication of approaching a closed shell, possibly at $N=184$ [18]. These observations go hand in hand with the increasing half-lives as $N=184$ is approached. On the other hand, there seems to be no effect at $N=172$, given by some calculations as the location of the shell closure (for a review, see e.g. Ref. [9]).

\subsubsection{Spontaneous fission}

The current understanding is that spontaneous fission will ultimately limit the number of chemical elements [1]. This is because of the long-range repulsive Coulomb interaction between the protons in the nucleus. In the region of SHN, there is a hindrance against fission caused by single-particle quantum effects which build up a fission barrier. Half-lives depend on the height and width of the barrier, on the existence of an outer barrier, and on the collective 
inertia of the nucleus [19-22]. Spontaneous fission is very much a dynamical problem, and calculation of the fission half-life is difficult. This is not helped by the fact that fission barriers are not observables that can be directly measured [20]. Once again, odd-mass and odd-odd nuclei are more difficult to treat than even-even nuclei. Very roughly, an odd nucleon increases the fission half-life by a few orders of magnitude.

Just like in $\alpha$ decay, systematic determination of spontaneous fission half-lives can give us information on shell and sub-shell closures. For the elements Fm and No, there is a clear maximum in the spontaneous fission half-life at $N=152$. At $\mathrm{Rf}$ there is a significant change in the behaviour of the half-life as a function of $N$, understood by the diminishing of the second barrier below the ground state [1]. The increase of the half-lives with $N$ for $\mathrm{Rf}, \mathrm{Sg}$ and Hs when $N$ approaches 162 is understood by the sub-shell nature of this neutron number. Likewise, the increase of the half-life by roughly two orders of magnitude when going from ${ }^{282} \mathrm{Cn}$ to ${ }^{284} \mathrm{Cn}$ and from ${ }^{286} \mathrm{Fl}$ to ${ }^{288} \mathrm{Fl}$ shows the influence of the predicted closed $N=184$ shell. The stability seems to increase more rapidly for spontaneous fission than for $\alpha$ decay when $N=184$ is approached.

\subsection{Mass measurements}

Mass measurements yield the nuclear binding energy without any knowledge about the nuclear state in question. Traditionally the masses of the heaviest nuclides have been obtained via $\alpha$-decay studies which give the energy released in the process. If the decay chain is long, the accumulated uncertainty can be large. If the nuclides in the chain are not of even-even type, the essential part of the decay scheme must be known which often is a challenge.

The state-of-the-art are Penning Trap Mass Spectrometry studies performed at GSI using SHIPTRAP. Mass excesses of $252,253,254,255$ No have been measured with uncertainties of $13-16 \mathrm{keV}$ [5]. Detection rates of on the order of one particle per minute are sufficient.

\subsection{X-ray identification}

The products of hot fusion reactions lack connection to previously known nuclei with firmly established proton and neutron numbers [1]. One possible way of providing such unambiguous information is to observe $X$ rays of the daughter nuclide in coincidence with the $\alpha$ decay of the parent [23]. First candidates for such a $Z$ determination were recently observed in decay chains of element 115 produced in the ${ }^{48} \mathrm{Ca}+{ }^{243} \mathrm{Am}$ reaction [24]. Although photons with energies compatible with the calculated energies of $\mathrm{Bh} K$ - $X$-rays were observed, statistics were not sufficient for completely excluding e.g. $\gamma$ rays as the source of these photons (see also Ref. [25]).

\section{Production}

The only successful method for producing SHN is heavy-ion induced fusion evaporation [6]. Two varieties have been used during the past few decades: In what is called cold fusion, lead and bismuth nuclei are bombarded with the most neutron rich naturally occurring isotopes of $\mathrm{Ti}, \mathrm{Cr}, \mathrm{Fe}, \mathrm{Ni}$ and $\mathrm{Zn}$ to make isotopes of $\mathrm{Rf}$ to element 113 . The produced isotopes are rather neutron deficient which means their neutron separation energies, $B_{\mathrm{n}}$, are high and fission barriers, $B_{\mathrm{f}}$, are low. Both features diminish the production cross section. Also, the half-lives are short because of the long distance to the beta stability line. The idea for cold fusion came from Oganessian [26]. In hot fusion reactions, man-made isotopes such as ${ }^{244} \mathrm{Pu}$, ${ }^{248} \mathrm{Cm}$ and ${ }^{249} \mathrm{Cf}$ are bombarded with the rare isotope ${ }^{48} \mathrm{Ca}$ [1]. The terms cold and hot refer to the excitation energy of the compound nucleus. Because of the high binding energy of 
${ }^{208} \mathrm{~Pb}$ and ${ }^{209} \mathrm{Bi}$, the excitation energy of the compound system in cold fusion is low, typically $15 \mathrm{MeV}$, and the compound system gets rid of its excitation energy by the evaporation of only one neutron. In hot fusion, the excitation energy is typically $35-40 \mathrm{MeV}$ and evaporation of three to five neutrons is normally observed.

There is a significant difference in the Coulomb energy which is proportional to the product $Z_{1} \cdot Z_{2}$ between cold and hot fusion reactions: If we compare the reactions ${ }^{76} \mathrm{Ge}+{ }^{208} \mathrm{~Pb}$ and ${ }^{48} \mathrm{Ca}+{ }^{244} \mathrm{Pu}$ to make element 114 we find a difference of eight in the neutron number of the compound nucleus. Because of the Coulomb hindrance to fusion, this means a factor of five to six orders of magnitude in compound nucleus formation [1]. The cold fusion reaction cross section goes down by a factor of eight orders of magnitude when going from No to element 113 [6]. Consequently, spherical SHN seem to be impossible to reach using cold fusion reactions. The main drawback using hot fusion reactions are the fission losses in the long evaporation chain. In each evaporation step, fission competes and typically wins by a factor of 10 or so.

Calculation of the probability, or cross section, for producing evaporation residues, results of neutron evaporation, is very difficult. It is also difficult to compare results of calculations with experimental results, especially for the process leading to the formation of the compound system. This is due to the difficulty of identifying products from competing processes such as fission and so called quasi fission. Also, because of the very small yields, the statistical uncertainty of the evaporation residue cross sections is high.

There are several methods for calculating evaporation residue cross sections [2, 27-29]. In the following, use is made of especially the review article of Zagrebaev and Greiner [2].

The process leading to the successful formation of the evaporation residue is normally divided into three stages. This division is justified by the different time scales of the phases. The three stages are

- The nuclei overcome the Coulomb barrier (termed contact or capture).

- The system evolves into a (spherical) compound nucleus (fusion).

- The compound nucleus cools down by neutron emission (survival).

The sub-barrier energy region is important during the contact stage. Channel coupling of the relative motion of the two nuclei with internal degrees of freedom (vibration, rotation) can enhance the cross section by orders of magnitude. This stage is relatively well understood, and accuracy of predictions is typically within a factor of two to three. Even this is, of course, undesirable from the point of view of planning experiments [30].

Compound nucleus formation is the least well understood reaction stage, mainly because of the large probability of quasi-fission [29]. There are significant differences in the theoretical treatment of the transformation from two nuclei to the compound nucleus as to how the nuclei lose their individuality [31].

Calculation of the survival stage is based on the statistical model. Typically, fission, neutron evaporation and $\gamma$ emission are taken into account. Fission is the dominating factor, and calculation of the fission width and in particular the fission barrier is the most uncertain part of the model. The value of the shell-damping parameter which takes into account the weakening of the shell effect with increasing excitation energy is especially important for SHN. The decisive factor in survival is the ratio of the neutron evaporation width to the fission width $\Gamma_{\mathrm{n}} / \Gamma_{\mathrm{f}}=\exp \left(B_{\mathrm{f}}-B_{\mathrm{n}}\right) / T$ where $T$ is the nuclear temperature.

It is worth noting that when the calculations of all three stages are combined, different theories may yield similar final evaporation residue cross sections but there may be significant differences in the probabilities for the individual stages [30]. 
As discussed in detail in Ref. [2], some rather general observations can be made when comparing cold and hot fusion reactions:

- Contact cross sections are roughly a factor of ten higher for hot fusion.

- The hot fusion probability is usually much higher than the cold fusion probability. Moreover, the cold fusion probability decreases much faster with increasing compound system proton number.

- The survival probability for the three-neutron evaporation channel (hot fusion) is much smaller than the survival probability of the one-neutron evaporation channel (cold fusion).

To put this on a quantitative level, here are some experimental evaporation residue cross sections and predictions:

- The lowest measured cross section is $\sim 20 \mathrm{fb}$ for the production of element 113 in cold fusion in the reaction ${ }^{70} \mathrm{Zn}+{ }^{209} \mathrm{Bi}$ [32].

- The cross section for the production of element 118 in the hot fusion reaction ${ }^{48} \mathrm{Ca}+{ }^{249} \mathrm{Cf}$ is $\sim 0.5 \mathrm{pb}[12]$.

- There are no positive results on element 119 or 120 [1, 33, 34]. The cross section limits for element 120 are on the order of $0.1-1 \mathrm{pb}$.

- One of the most promising candidates for the production of element 120 is the reaction ${ }^{50} \mathrm{Ti}+{ }^{249} \mathrm{Cf}$. Two representative cross-section predictions are $40 \mathrm{fb}$ [35] and $6 \mathrm{fb}$ [36].

\section{Conclusion}

The amount of information which has been gathered during the past ten to fifteen years both in the No region and on the heaviest elements is extensive. Rather detailed level schemes have been built for certain nuclei in the No region, and intriguing structure details especially from $\alpha-\gamma$ coincidence measurements have emerged for even heavier nuclei. The influence of the $Z=108$ and $N=152,162$ shells has been firmly established and there are clear indications of a closed neutron shell at higher $N$, possibly $N=184$. Firm identification of hot fusion products by $X$-ray methods seems to be a possibility in the near future in selected cases.

Useful discussions with M. Bender, P.T. Greenlees, R.-D. Herzberg, F.P. Heßberger, T.L. Khoo and A. Sobiczewski are gratefully acknowledged.

\section{References}

[1] Yu.Ts. Oganessian and V.K. Utyonkov, Nucl. Phys. A944, 62 (2015).

[2] V.I. Zagrebaev and W. Greiner, Nucl. Phys. A944, 257 (2015).

[3] M. Asai, F.P. Heßberger, A. Lopez-Martens, Nucl. Phys. A944, 308 (2015).

[4] Ch. Theisen, P.T. Greenlees, T.-L. Khoo, P. Chowdhury, T. Ishii, Nucl. Phys. A944, 333 (2015).

[5] M. Block, Nucl. Phys. A944, 471 (2015).

[6] Yu.Ts. Oganessian and V.K. Utyonkov, Rep. Prog. Phys. 78, 036301 (2015).

[7] A. Sobiczewski, Radiochim. Acta 99, 395 (2011).

[8] S. Hofmann, J. Phys. G 42, 114001 (2015).

[9] M. Bender and P.-H. Heenen, J. Phys. Conf. Series 420, 012002 (2013). 
[10] R.R. Chasman, I. Ahmad, A.M. Friedman, J. Erskine, Rev. Mod. Phys. 49, 833 (1977).

[11] G. Münzenberg, Nucl. Phys. A944, 5 (2015).

[12] Yu.Ts. Oganessian et al., Phys. Rev. Lett. 109, 162501 (2012).

[13] R.-D. Herzberg and P. T. Greenlees, Prog. Part. Nucl. Phys. 61, 674 (2008).

[14] R.-D. Herzberg et al., Nature 442, 896 (2006).

[15] G.D. Dracoulis, Phys. Scr. T152, 014015 (2013).

[16] P.T. Greenlees et al., Phys. Rev. C 78, 021303(R) (2008).

[17] A.N. Andreyev et al., Phys. Rev. Lett. 110, 242502 (2013).

[18] V.K. Utyonkov et al., Phys. Rev. C 92, 034609 (2015).

[19] M. Kowal, P. Jachimowicz, A. Sobiczewski, Phys. Rev. C 82, 014303 (2010).

[20] A. Staszczak, A. Baran, W. Nazarewicz, Phys. Rev. C 87, 024320 (2013).

[21] B.S. Bhandari and Y.B. Bendardaf, Phys. Rev. C 45, 2803 (1992).

[22] R. Smolańczuk, J. Skalski, A. Sobiczewski, Phys. Rev. C 52, 1871 (1995).

[23] C.E. Bemis et al., Phys. Rev. Lett. 31, 647 (1973).

[24] D. Rudolph et al., Phys. Rev. Lett. 111, 112502 (2013).

[25] J.M. Gates et al., Phys. Rev. C 92, 021301(R) (2015).

[26] Yu.Ts. Oganessian, Lect. Notes Phys. 33, 221 (1975).

[27] G.G. Adamian, N.V. Antonenko, W. Scheid, V.V. Volkov, Nucl. Phys. A627, 361 (1997).

[28] W.J. Świątecki, K. Siwek-Wilczyńska, J. Wilczyński, Phys. Rev. C 71, 014602 (2005).

[29] G. Giardina et al., J. Phys. Conf. Series 282, 012006 (2011).

[30] W. Loveland, Eur. Phys. J. A 51, 120 (2015).

[31] V.I. Zagrebaev, Y. Aritomo, M.G. Itkis, Yu.Ts. Oganessian, M. Ohta, Phys. Rev. C 65, 014607 (2001).

[32] K. Morita et al., J. Phys. Soc. Jpn 81, 103201 (2012).

[33] Yu.Ts. Oganessian et al., Phys. Rev. C 79, 024603 (2009).

[34] S. Hofmann et al., Eur. Phys. J. A 52, 180 (2016).

[35] V.I. Zagrebaev and W. Greiner, Phys. Rev. C 78, 034610 (2008).

[36] K. Siwek-Wilczyńska, T. Cap, M. Kowal, A. Sobiczewski, J. Wilczyński, Phys. Rev. C 86, 014611 (2012). 